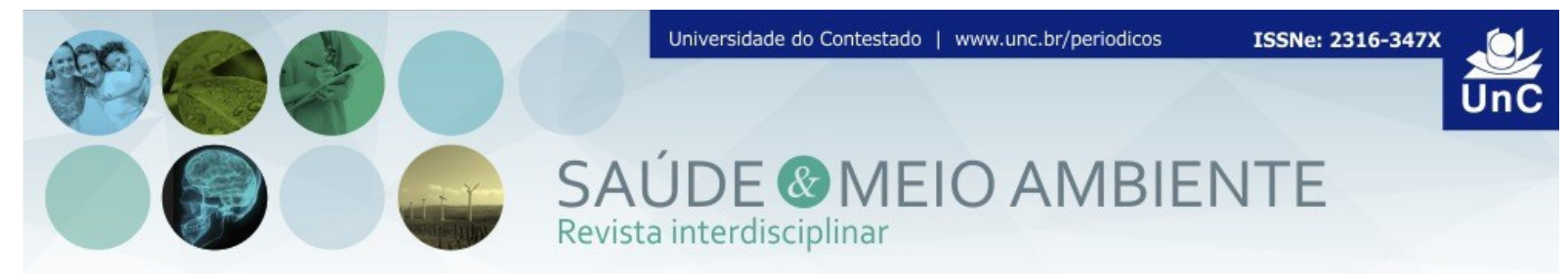

\title{
AVALIAÇÃO DA REALIZAÇÃO DO TESTE RÁPIDO NA CONSULTA DE ENFERMAGEM COMO ENFRENTAMENTO DA SÍFILIS
}

\section{EVALUATION OF THE PERFORMANCE OF THE QUICK TEST IN NURSING CONSULTATION AS FACING SYPHILIS}

\author{
Eliz Cristine Maurer Caus ${ }^{1}$ \\ Jéssica Angelita de Andrade ${ }^{2}$
}

\begin{abstract}
RESUMO
INTRODUÇÃO: A Sífilis é uma Infecção Sexualmente Transmissível (IST), de alta prevalência na atualidade. Nesse contexto, destaca-se o potencial da consulta de Enfermagem como estratégia para redução da incidência e consequências para a saúde humana. OBJETIVOS: Avaliar a realização do Teste Rápido - TR para Sífilis na consulta de enfermagem na prevenção, diagnóstico precoce e tratamento da sífilis no município de Mafra/SC. MATERIAIS E MÉTODOS: Trata-se de uma pesquisa de campo, quali-quantitativa, na qual coletou-se em livro de registro dos últimos 5 anos os resultados dos TRs de sífilis; e aplicou-se uma questão aberta à 19 enfermeiros da atenção básica e vigilância epidemiológica. RESULTADOS: A cobertura de TRs de 2015 a julho de 2019 foi de 18,27\% da população geral; notificou-se 1,07\% (306) de casos positivos, sendo a maioria do sexo masculino e jovens na faixa etária de 15 a 24 anos. Os TRs são ofertados durante consulta de enfermagem, no programa de detecção precoce do câncer cérvico uterino, no pré-natal usuários de maior vulnerabilidade (presidiários), no acolhimento à demanda espontânea. Utilizam a estratégia de divulgação por meio do ACS, nos grupos organizados, folder/cartazes na unidade, PSE, eventos na comunidade/externo em prol ao outubro rosa/novembro azul. CONCLUSÕES: Ao longo dos anos, de 2015 a 2019 percebe-se o aumento significativo de oferta e procura dos usuários ao TR da sífilis, mas ressalta-se a otimização dos recursos por meio de um rastreamento organizado.
\end{abstract}

Palavras-Chave: Enfermagem. Consulta. Sífilis.

\begin{abstract}
INTRODUCTION: Syphilis is a sexually transmitted infection (STI), highly prevalent today. In this context, the potential of nursing consultation as a strategy for reducing the incidence and consequences for human health is highlighted. OBJECTIVES: To evaluate the performance of the rapid test for Syphilis in the nursing consultation in the prevention, early diagnosis and treatment of syphilis in the municipality of Mafra / SC. \footnotetext{
Contestado. Campus Mafra. Santa Catarina. Brasil. E-mail: madonaleita@bol.com.br

${ }^{2}$ Acadêmica de Enfermagem, bolsista da iniciação científica Art. 171. Universidade do Contestado. Campus Mafra. Santa Catarina. Brasil. E-mail: jeh angelita@hotmail.com
}

1Docente do curso de enfermagem, orientadora da iniciação científica do Art. 171. Universidade do
\end{abstract}


MATERIALS AND METHODS: This is a field research, quali-quantitative, in which the results of rapid syphilis tests were collected in a record book from the last 5 years; and an open question was applied to 19 nurses in primary care and epidemiological surveillance. RESULTS: Rapid test coverage from 2015 to jul. 2019 was $18.27 \%$ of the general population; $1.07 \%$ (306) of positive cases were reported, with the majority being male and young people aged 15 to 24 years. Rapid tests are offered during a nursing consultation, in the early detection program for cervical cancer of the uterus, in prenatal users who are most vulnerable (prisoners), in welcoming spontaneous demand. They use the dissemination strategy through the ACS, in organized groups, folder / posters in the unit, PSE, community / external events in favor of the pink October / blue November. CONCLUSIONS: Over the years, from 2015 to 2019 there has been a significant increase in the supply and demand of users to the rapid syphilis test, but the optimization of resources is emphasized through organized tracking.

Keywords: Nursing. Query. Syphilis.

\section{INTRODUÇÃO}

O termo sífilis teve origem de um poema escrito pelo médico e poeta Girolamo Fracastoro, de 1530, em seu livro intitulado Syphilis Sive Morbus Gallicus ("A sífilis ou mal gálico"). Nela, o autor narra a história de Syphilus, um pastor que recebeu um castigo após amaldiçoar o deus Apolo, e em 1546, levanta a hipótese de que a doença fosse transmitida na relação sexual. Nessa época, essa ideia não foi levada em consideração e, somente após 300 anos, com Louis Pasteur, passou a ter crédito. ${ }^{1}$

A sífilis é causada pelo Treponema pallidum, descoberto 1905, pode afetar praticamente todos os órgão e sistemas, possui tratamento eficaz, de baixo custo e disponível nas unidades de saúde, no entanto, ainda é um grave problema de saúde pública, principalmente pelo fato da via ser sexual (sífilis adquirida), mas ainda há verticalmente (sífilis congênita) da mãe para o filho. ${ }^{2}$

Pode-se afirmar que a sífilis é de fácil prevenção, porém ainda apresenta crescente número de casos tanto na esfera municipal, estadual como Federal, e demonstra grande dificuldade de prevenção na população adulto/jovem. ${ }^{3}$

Para enfrentamento na condução das ações dos profissionais de saúde perante os casos de sífilis, forma criadas ferramentas essenciais que usarão de fluxogramas e algorítmos para as Infecções Sexualmente Transmissíveis - ISTs, abordando as etapas para o acolhimento da pessoa. Também utilizado da abordagem sindrômica qual extrema objetividade, que no momento do acolhimento será coletado as queixas e dados, sendo oferecido tratamento e diagnostico em tempo oportuno, resultando em um melhor atendimento na atenção básica de saúde. ${ }^{3}$

A Sífilis é uma doença infectocontagiosa sistêmica, de evolução crônica, com manifestações cutâneas temporárias, provocadas por uma espiroqueta. Sua evolução é dividida em recente e tardia. A transmissão da Sífilis Adquirida é 
sexual, na área genitoanal, na quase totalidade dos casos. Na Sífilis Congênita, há infecção fetal via hematogênica, em qualquer fase gestacional ou estagio clínico da doença materna. A transmissão por transfusão sanguínea é rara nos dias atuais ${ }^{4}$.

Dessa forma, a Sífilis adquirida e a sífilis congênita devem ser notificadas em ficha específica individual, no Sistema de Informação de Agravos de Notificação SINAN, que foi instituída por meio da portaria $n^{0} 2.472 / 2010 .^{56}$

A distribuição da sífilis adquirida é mundial e a estimativa no ano de 2012 foi de 5,6 milhões casos novos ${ }^{7}$. No Brasil, após uma série de aumentos de sífilis adquirida, congênita e gestacional, foram apresentados pela primeira vez os dados de sífilis adquirida no Boletim Epidemiológico, numa série histórica de 2010 feitas por meio do SINAN. O controle da sífilis tem se tornado um desafio para a saúde pública, o que se nota pelas notificações no SINAN nos anos de 2010 a 2016, onde obtiveram um total de 227.663 casos de sífilis adquirida ${ }^{8}$.

A resolução COFEN 544/2017, aborda a consulta de enfermagem como ato privativa do enfermeiro, na qual o enfermeiro utiliza o método científico para identificar problemas no processo saúde/doença de um indivíduo, prescreve e implementa cuidados de Enfermagem que contribuam para a promoção, prevenção, proteção da saúde, recuperação e reabilitação do mesmo, da família e da comunidade ${ }^{9}$.

O estímulo à realização da testagem rápida tem sido considerado uma das estratégias para prevenção da transmissão da sífilis e diminuição da morbidade e mortalidade. A testagem possibilita o diagnóstico precoce, início do tratamento em tempo oportuno e a manutenção de uma alta adesão à terapia antimicrobiana.

O enfermeiro tem o desafio de implementar o cuidado em enfermagem na construção de relações interpessoais de diálogo, escuta, humanização e respeito, pois o vínculo com o(a) usuário(a) é importantíssimo para adesão ao tratamento adequado, bem como na abordagem ao(a/as/aos) parceiro(a/as/os).$^{10}$

Assim, a comunicação aos parceiros(as) sexuais são aspectos importantes a serem abordados pelos profissionais de enfermagem durante o aconselhamento, nas ações de educação e identificação de casos de IST mediante testagens rápidas. ${ }^{11}$ Ademais, recomenda-se para atenção integral às pessoas com IST e suas parcerias sexuais no Brasil que os profissionais estejam aptos a exercer a prevenção individual e coletiva; oferta de diagnóstico das IST assintomáticas; manejo de IST sintomáticas com fluxogramas com/sem laboratório; abordagem às parcerias sexuais e notificação epidemiológica dos casos. ${ }^{12}$

Dessa forma, traçou-se o seguinte objetivo geral: Avaliar a realização do teste rápido para Sífilis na consulta de enfermagem na prevenção, diagnóstico precoce e tratamento da sífilis no município de Mafra/SC. 


\section{MATERIAL E MÉTODOS}

Trata-se de uma pesquisa de campo do tipo quali-quantitativa, cuja coleta de dados se deu em base documental e entrevista. Dessa forma, coletou-se informações por meio do livro registro da unidade e/ou bloco de laudo do resultado dos testes rápidos de 2015 à julho de 2019, utilizando-se um roteiro, com as seguintes informações de interesse; idade, sexo, resultado do teste rápido de sífilis.

Para os dados qualitativos foi aplicado e as seguintes questões abertas as(aos) enfermeiras(os): quais estratégias você utiliza para ampliar a oferta de TRs aos usuários? Caso não utilize nenhuma estratégia como oferece o tratamento ao usuário?

A pesquisa no livro de registro e o questionário foi aplicado pela própria pesquisadora, na unidade básica de saúde à 9 enfermeiros que atuam no modelo Estratégia Saúde da Família - ESF e ao enfermeiro que atua na vigilância epidemiológica, de um município do Planalto Norte Catarinense. O município do estudo possui 18 unidades básica de saúde no modelo ESFs abrangendo $100 \%$ de cobertura da população, sendo a amostra composta por $50 \%$ do total de enfermeiros.

A coleta de dados foi realizada no mês de agosto de 2019, por isso obteve-se os dados até julho de 2019, em seguida os mesmos foram organizados em planilha EXCEL e apresentados na forma gráfica. As respostas dos questionários foram organizadas conforme a similaridade e singularidade e analisadas à luz do referencial bibliográfico.

\section{RESULTADOS}

A cobertura de teste rápidos realizados entre 2015 a 2019 foi de $18,27 \%$ da população geral, os casos notificados nesse período foram de 1,07\% (306), sendo incluso todos os casos de testes rápidos para sífilis reagentes, independente em ser gestante e da fase em que a doença se encontre. A sífilis congênita não consta nos dados, pois o diagnóstico é realizado a nível hospitalar na assistência ao neonato.

O gráfico a seguir mostra a evolução do número de testes rápidos da sífilis realizada pelas enfermeiras das Unidades Básicas de Saúde (UBSs) do município: 
Gráfico 1 - Série histórica do número absoluto de TR da Sífilis realizados de 2015 a julho de 2019

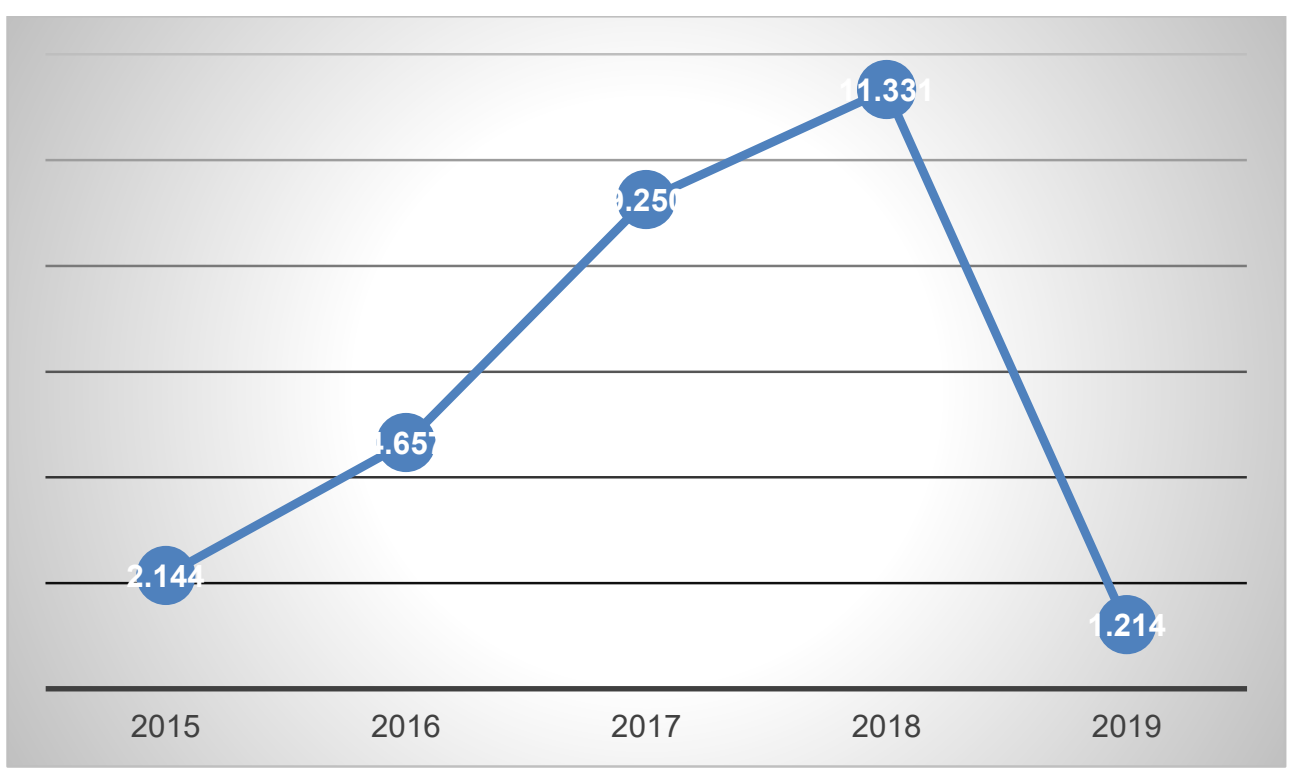

Fonte: Dados da pesquisa (2019)

O gráfico 2 se refere ao percentual de testes reagentes em relação ao total de exames realizados, apontando a otimização deste recurso e consequente efetividade nas ações de intervenção para interrupção na cadeia epidemiológica de transmissão da doença.

Gráfico 2 - Série histórica do número absoluto de casos de sífilis adquirida notificados a partir do TR, 2015 a julho de 2019

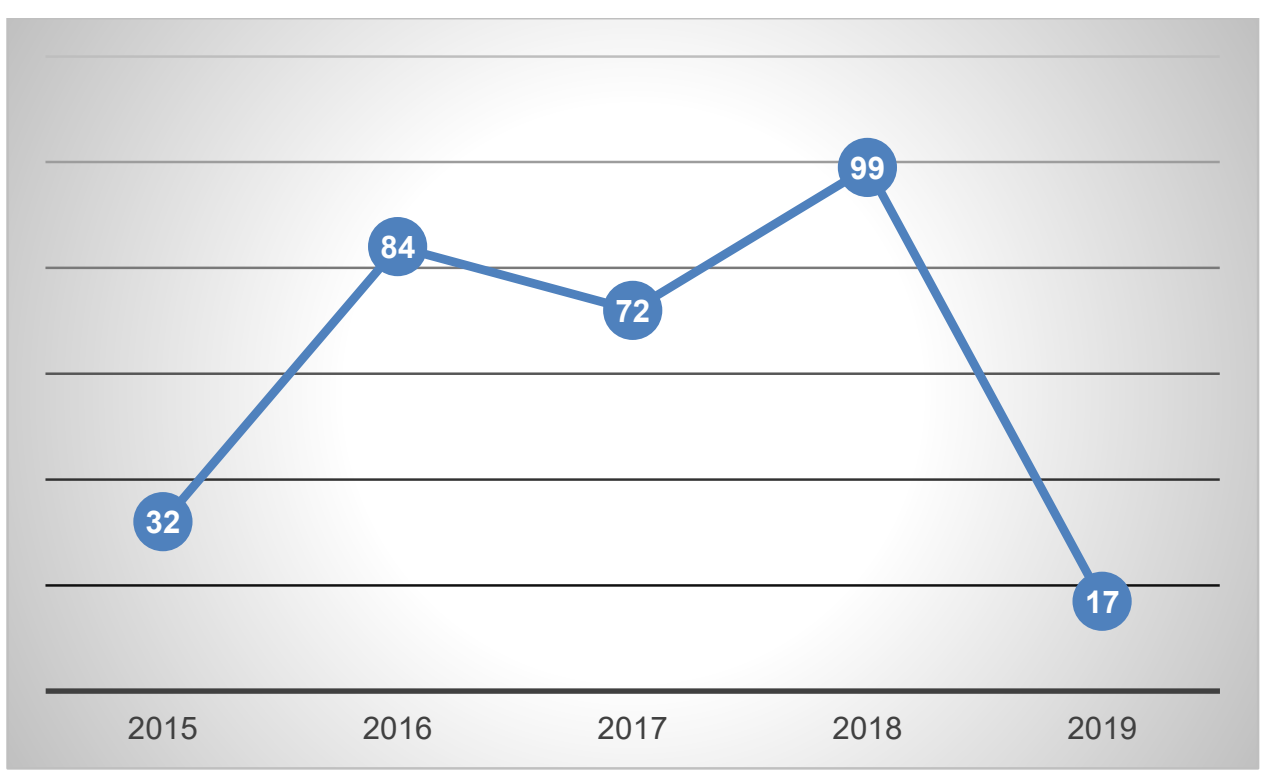

Fonte: Dados da pesquisa (2019) 
O gráfico abaixo se refere à relação entre o número de testes rápidos da sífilis positivos pelo número total de testes realizados nos serviços de saúde.

Gráfico 3 - Proporção de TR reagentes de Sífilis em relação ao total de exames realizados nos anos de 2015 a julho de 2019

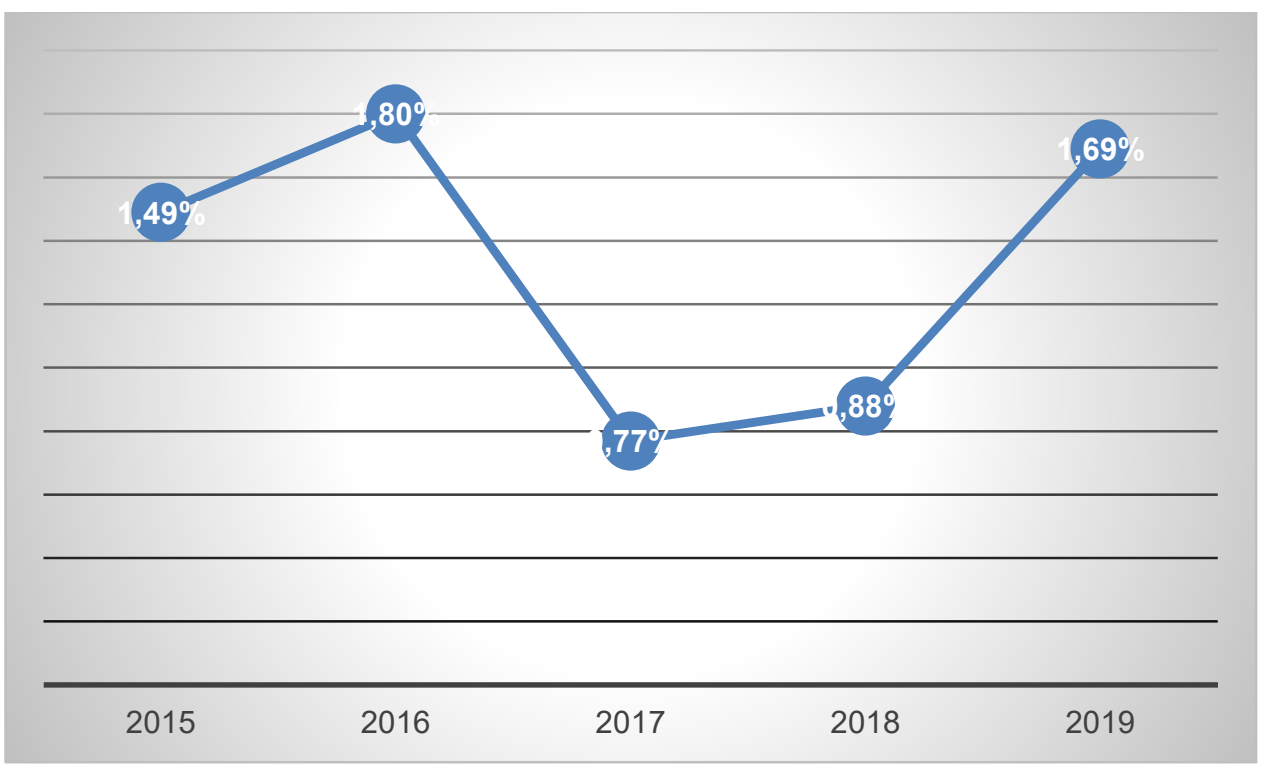

Fonte: Dados da pesquisa (2019)

O gráfico 4 se refere ao perfil demográfico dos indivíduos que tiveram resultado reagente no teste rápido da sífilis, na qual em 2018 foram registrados 99 casos de sífilis adquirida. Considerando os dados de 81 indivíduos pois em 18 casos não havia identificação de sexo, idade e condição obstétrica. Dessa forma, a faixa etária de maior prevalência foi de 15 a 39 anos, representando $65,7 \%$. 
Gráfico 4 - Número absoluto de TR de Sífilis positivos por faixa etária X sexo e condição de gestação em 2018

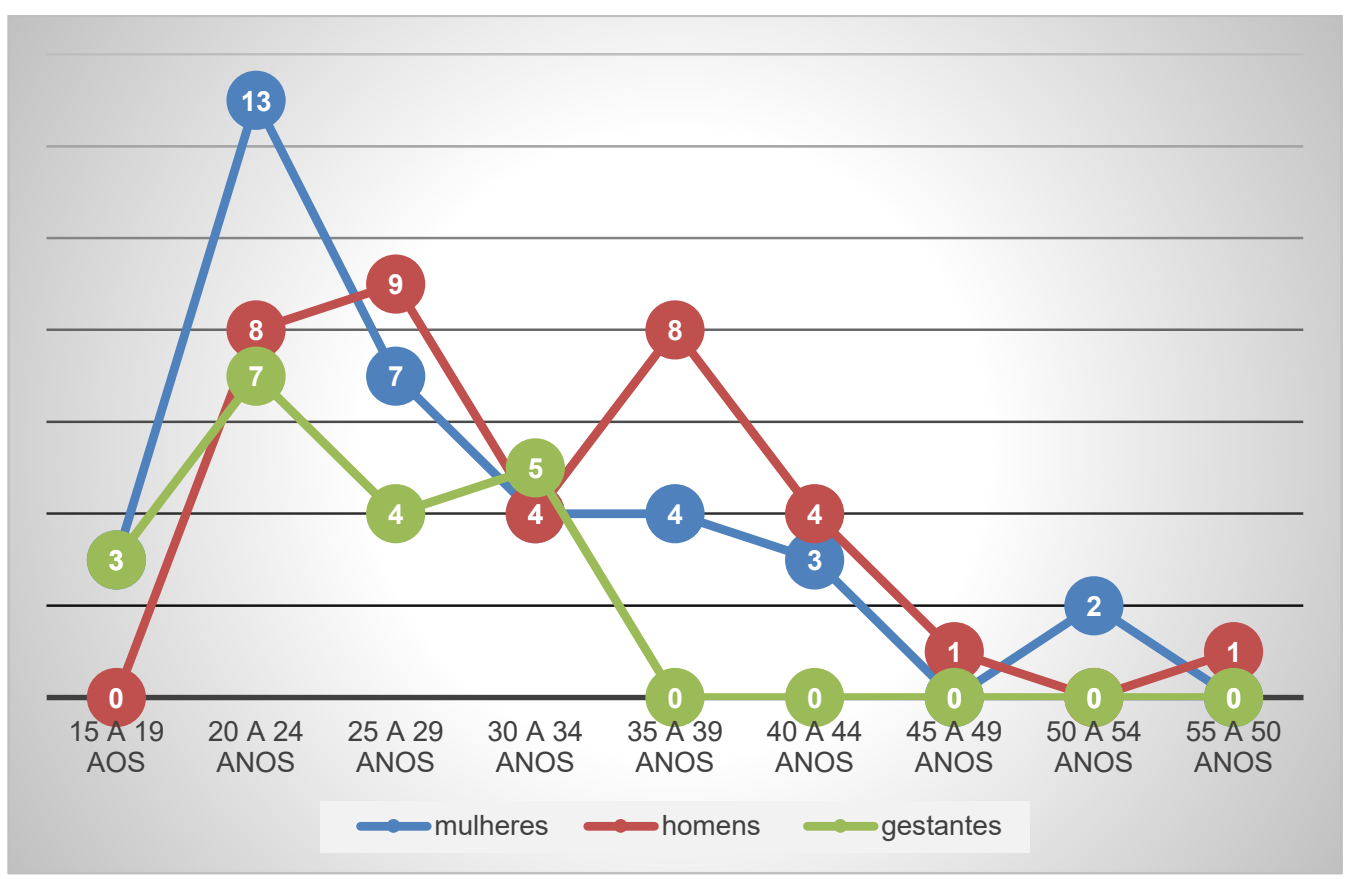

Fonte: Dados da pesquisa (2019)

O gráfico 5 aponta que no primeiro semestre de 2019, foram detectados 05 casos em gestantes, $07 \mathrm{em}$ mulheres e $05 \mathrm{em}$ homens e a faixa etária de maior prevalência foi de 15 a 39 anos.

Gráfico 5 - Número absoluto de TR da Sífilis positivos por faixa etária X sexo e condição de gestação de 01 a $07 / 2019$

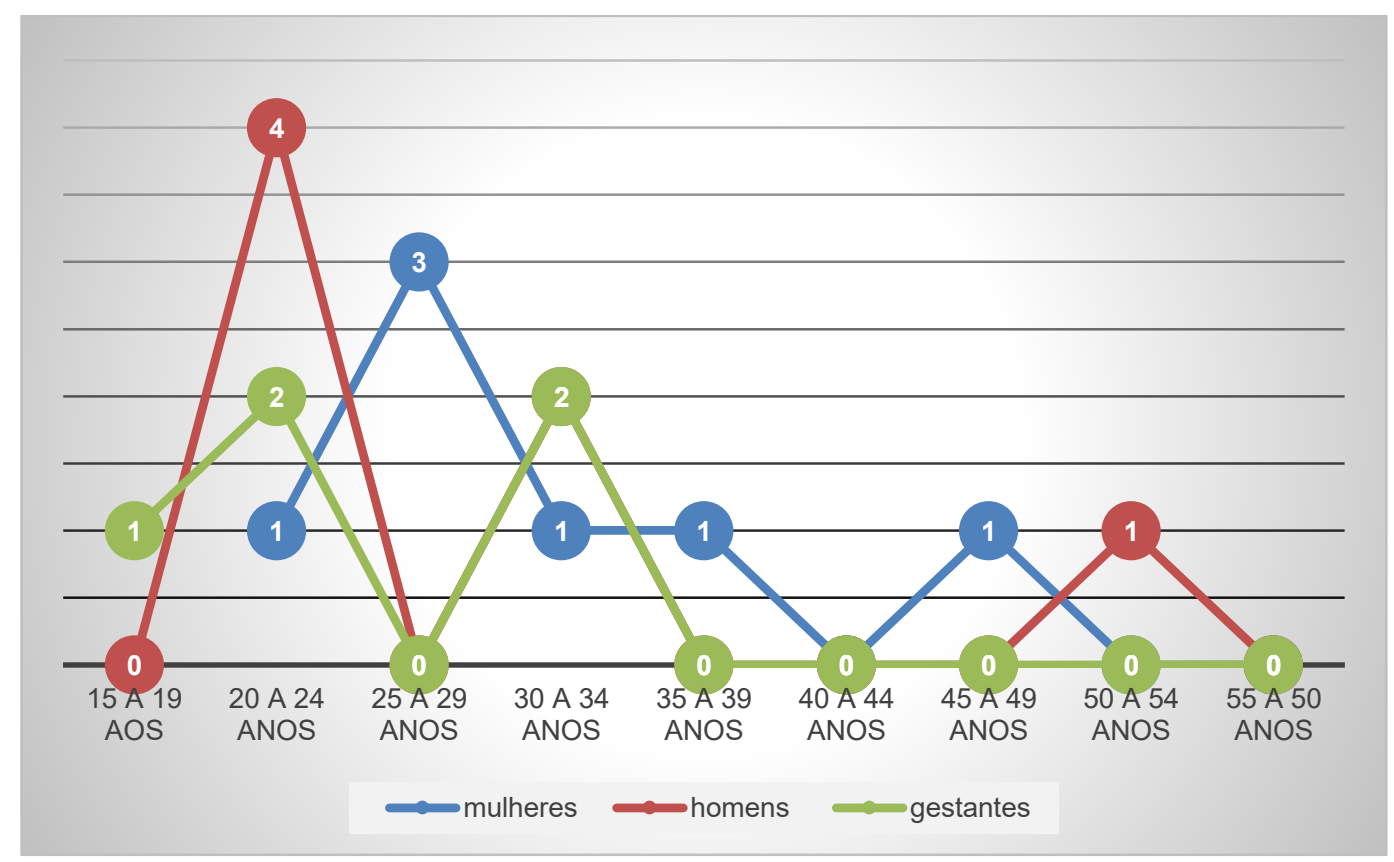

Fonte: Dados da pesquisa (2019) 
O gráfico abaixo apresenta as formas de atuação do enfermeiro quanto à abordagem na consulta de enfermagem e na divulgação para realização do teste rápido da sífilis.

Gráfico 6 - Meios de divulgação utilizados pelos enfermeiros para enfrentamento da Sífilis/2019

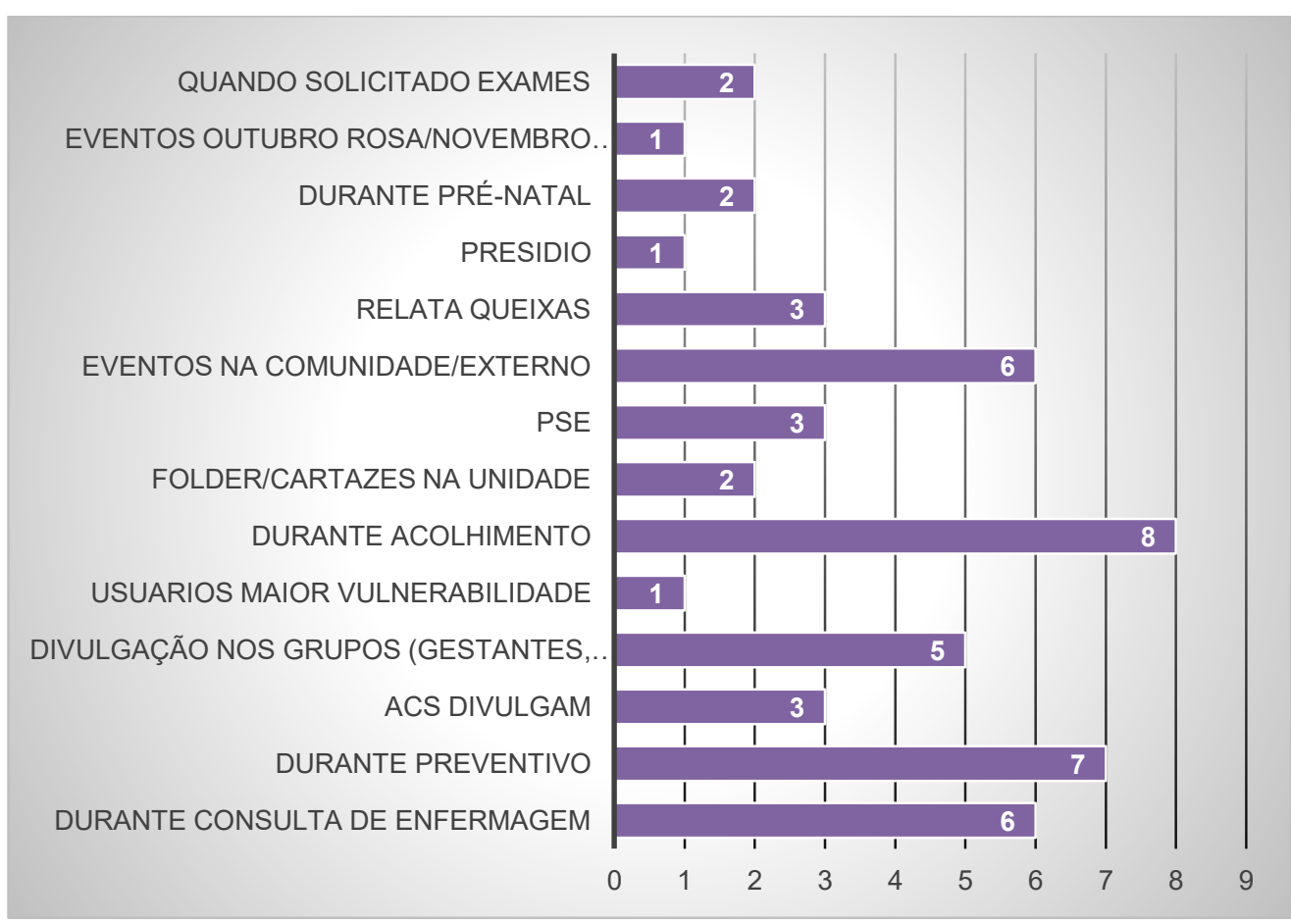

Fonte: Dados da pesquisa (2019)

\section{DISCUSSÃO}

Em relação à evolução do quantitativo de TRs realizados de 2015 a julho de 2019, deve-se considerar que se refere somente ao primeiro semestre de 2019, mas afere-se que proporcionalmente apresenta um decréscimo na oferta do teste, o que se justifica pelo desabastecimento do kit de TR da sífilis pelo Ministério da Saúde ao estado e municípios catarinenses. Diante do crescente aumento da oferta dos TRs percebe-se a melhoria do serviço, seja na organização do processo de trabalho, número de profissionais adequados e qualificados para tal função de forma a oferecer um cuidado integral à saúde dos usuários.

Observa-se um aumento na detecção de testes reagentes para sífilis, exceto de 2019 na qual o sistema de saúde sofreu desabastecimento pela falta do reagente do teste, repercutindo na oferta do serviço aos usuários.

No município do estudo, a exemplo de outros municípios, estados e países, a sífilis vem aumentando sua incidência e segundo o boletim epidemiológico, a sífilis 
adquirida apresentou aumentou de 2,0 casos por 100 mil habitantes em 2010 para 58,1 casos por 100 mil habitantes em 2017, no país como um todo. No entanto, desse todo, $29.169(24,3 \%)$ são da Região Sul. ${ }^{13}$

Quanto a proporção entre o número de testes rápidos da sífilis positivos pelo número total de testes realizados nos serviços de saúde, percebe-se que em 2015 foram realizados 2.144 exames e 19 resultados deram positivos, em uma proporção de 1,49\%; em 2016 foram 4.657 para 84 positivos com 1,8\%; em 2017 foram em 9250 para 72 positivos, com 0,77\%; em 2018 em um total de 11.331 para 99 positivos, com 0,88\%; e em 2019, considerando apenas os meses investigados tem-se 1.214 para 17 casos novos perfazendo $1,69 \%$ de positividade, ou seja, nem sempre o maior número de exames realizados representa maior número de casos novos encontrados, o que reforça a importância de se realizar um rastreamento organizado, baseado em critérios e protocolos de atendimento.

O rastreamento organizado se constitui especialmente importante nos países onde os recursos financeiros são escassos e insuficiente para a saúde pública. $\mathrm{O}$ rastreamento quando apropriado e estabelecido em um programa organizado, permite uma detecção precoce da doença assegurando o cidadão uma atenção à saúde de qualidade. ${ }^{14}$

Em 2018 quanto ao perfil demográfico e condição obstétrica, percebe-se que a faixa etária de maior prevalência foi de 15 a 39 anos, representando $65,7 \%$ desta, ou seja, são indivíduos jovens em fase plena da atividade sexual, exposta a contrair e transmitir ISTs, sem as devidas medidas profiláticas, ou seja, tratamento correto e uso de preservativos e informações adequadas. Os resultados indicam a vulnerabilidade dos jovens às IST podendo ser por início precoce da atividade sexual, a necessidade de aceitação e inserção em grupos sociais, aumento do consumo de álcool e outras drogas, e questões de gênero, entre outros.

No estudo realizado por Souza, em 2017, sobre o uso sistemático do preservativo por adolescentes, relata que 46\% usam "sempre"; 45\% "algumas vezes", e $9 \%$ referem que "nunca" utilizam o preservativo em suas relações sexuais. Mostrando que por mais que ainda haja o cuidado, a transmissão sexual de infecções pode ocorrer pelo fato de desconhecerem os métodos de prevenção e contágio, ou não atribuírem a devida importância à situação por considerarem que há tratamento disponível. Dessa forma, é fundamental o papel educativo que o profissional de enfermagem representa na promoção da saúde dos adolescentes. ${ }^{15}$

Em um estudo Baiano, a incidência de sífilis adquirida teve como faixa etária predominante, entre 15 e 35 anos $^{16}$. O que também é semelhante aos dados apresentados no Boletim Epidemiológico do ano de 2016, onde, no Brasil, 55,6\% dos casos de sífilis adquirida em 2015 eram na faixa etária de 20-39 anos. ${ }^{17}$ Já em Feira de Santana no período de 2003 a 2012, a prevalência maior foi apresentada em adolescentes entre 11 e 20 anos. $^{18}$ Assim sendo, com base em todos os outros estudos em consonância com o atual, sugere-se que a vida sexual esteja iniciando precocemente, trazendo consigo consequências, dentre elas uma exposição maior de risco de contaminação de infecções sexualmente transmissíveis. Além disso, é importante ressaltar que a vida sexual nessa faixa etária é mais ativa. ${ }^{19}$ 
Quanto ao aumento da demanda da procura dos TRs é importante salientar que sem a necessidade de agendamentos e resultado em poucos minutos faz com haja uma maior procura pelos usuário masculino para o exame, além de que a mulher com resultado positivo notifica e encaminha seu(s) sua(s) parceiro(s) parceira(s) e vise versa ao serviço de saúde. Só acontecerá a interrupção do ciclo de transmissão se ambos os parceiros forem tratados, sendo realizado a notificação de parceiros sexuais, pois realizada corretamente reduz a infecção persistente do paciente-índice, identifica as infecções sexuais assintomáticas, contribui para a redução da transmissão. ${ }^{20}$

Afere-se que a identificação de 15 gestantes na faixa etária entre 20 e 34 anos é de extrema importância e preocupação, pois é uma situação que vem crescendo no município e região, pois a sífilis congênita é uma condição grave que afeta a saúde e o futuro da criança. Em 2017, o número total de casos notificados no Brasil foi de 49.013 (28,4\% mais casos que no ano anterior), dos quais 7.864 (16\%) são da Região Sul. ${ }^{13}$ A prevalência da sífilis gestacional foi de $0,57 \%$, em um estudo realizado na $15^{a}$ Regional de Saúde do Estado do Paraná, no período de 2011 a 2015, demostrando que vem crescendo, apontando a necessidade de aumento de informações à população. ${ }^{21}$

A cadeia de transmissão da sífilis só será interrompida quando todos fizerem a sua parte, profissionais de saúde atendendo com competência, usuário com corresponsabilidade no tratamento, medidas preventivas, comunicação, diagnóstico e tratamento dos parceiros.

Os dados do primeiro semestre de 2019 apontam uma leve tendência ao aumento de casos de sífilis adquirida, pois proporcionalmente foram detectados, $1,69 \%$ de positividade nos TRs realizados neste período, sendo ultrapassado apenas por 2016 quando teve um percentual de 1,8\% apontando a necessidade de se mobilizar mais ações para informar a população e investigar precocemente essa doença que muitas vezes pode passar desapercebida.

Em relação ao perfil dos casos novos mantém-se a mesma caracterização, são jovens, acometem homens e mulheres em proporção semelhante e identifica a gestação como uma condição que deve ter prioridade.

Sabe-se que é por meio de um pré-natal de qualidade, com a realização do teste rápido da sífilis precocemente que se propicia a intervenção oportuna, já que existem fatores de risco associados à sua forma congênita. Dessa forma, é importante a realização de um pré-natal adequado, por profissionais capacitados e envolvidos com a assistência, permitindo o tratamento oportuno das mães e dos parceiros infectados, de maneira significativa, permitindo a ocorrência da sífilis congênita e prevenir as complicações graves dessa doença na criança a curto e longo prazo caso ela seja acometida durante a gestação. ${ }^{22}$

Quanto a forma de atuação do enfermeiro na abordagem educativa para realização do teste rápido da sífilis percebe-se que o TR é ofertado durante consulta de enfermagem, no programa de detecção precoce do câncer cérvico uterino, no prénatal, aos usuários de maior vulnerabilidade (presidiários), no acolhimento à demanda 
espontânea ao ouvir a queixa do usuário. Utilizam a estratégia de divulgação por meio dos Agentes Comunitários de Saúde, nos grupos organizados como HIPERDIA, por meio de folder e cartazes na unidade, em palestras no Programa Saúde do Escolar e em eventos na comunidade em prol ao outubro rosa/novembro azul.

Foi relatada por $88,8 \%$ dos enfermeiros que o acolhimento é um momento oportuno e sabe-se que este constitui oportunidade "ouro" para estabelecer vínculo entre o profissional e o usuário, e por meio de uma escuta qualificada promove-se a Sistematização da Assistência de Enfermagem (SAE).

Sente-se falta da citação dos protocolos de enfermagem como instrumento de cuidado nas ações de enfrentamento às ISTs e em especial à sífilis. O acolhimento é uma prática presente em todas as relações de cuidado, seja no ato de receber e escutar as pessoas, acolhendo a demanda espontânea que chega às unidades de atenção básica; é o ponto chave para identificação da vulnerabilidade podendo ofertar mais teste rápidos aumento sua cobertura. ${ }^{23}$

Outro método fundamental é a Sistematização da Assistência de Enfermagem (SAE) pois permite que enfermeiro realize a prestação de cuidados individualizados, podendo implementar o processo de enfermagem, utilizado do pensamento crítico e raciocínio clinico, necessitando do vínculo entre profissional e usuário, sendo realizada em cinco etapas sequenciais e inter-relacionadas, a saber: levantamento de dados, diagnóstico, planejamento, implementação e avaliação, podendo oferecer que se enxergue o paciente no seu todo. ${ }^{24}$

\section{CONCLUSÃO}

Com esta pesquisa pode-se concluir que os testes rápidos configuram em uma estratégia eficiente para detecção precoce de sífilis principalmente na gestação e assim intervir oportunamente, tratando e evitando a sífilis congênita. Como a sífilis é uma doença que traz graves consequências a saúde humana em sua fase terciária e principalmente quando acomete o feto é preciso prevenir a transmissão na população em geral.

Os resultados deste estudo constataram que a sífilis adquirida não atinge um grupo específico, apesar de ter apresentado um valor significativo em pessoas com idade entre 20-40 anos. O crescimento no número de casos de sífilis adquirida no território brasileiro vem aumentando consideravelmente, trazendo uma preocupação geral, pois, além dos problemas causados pela própria doença, ainda há o aumento do risco de infecção pelo HIV, reforçando a necessidade de medidas de controle direcionadas à informação à população, incentivo ao uso do preservativo e, principalmente, diagnóstico e tratamento dos pacientes e parceiro/as, na qual o enfermeiro tem papel imprescindível.

Dessa forma, a pesquisa aponta que o enfermeiro tem contribuído significativamente para o aumento da oferta de testes rápidos à população, pois ele é 
um profissional preparado para atuar nas ações preventivas, promocionais e curativas de saúde no que concerne as ISTs.

Os enfermeiros pesquisados utilizam as estratégias que possuem para o enfrentamento das ISTs, ou seja, por meio das atividades educativas, ações programáticas, campanhas e atendimento à demanda, no entanto há necessidade de valorizar os protocolos de enfermagem como instrumento de cuidado nas ações de enfrentamento às ISTs e em especial à sífilis.

Ao longo dos anos, de 2015 a 2019 percebe-se o aumento significativo de oferta e procura dos usuários ao teste rápido da sífilis, mas ressalta-se a otimização dos recursos por meio de um rastreamento organizado.

Dessa forma, considera-se que os objetivos da pesquisa em avaliar a realização do teste rápido para Sífilis na consulta de enfermagem na prevenção, diagnóstico precoce e tratamento da sífilis no município foram alcançados, apesar da dificuldade de se obter acesso aos dados diante do sigilo das informações.

AGRADECIMENTO: A equipe de saúde das ESFs do município e em especial do ESF CAIC, pelo auxilio na pesquisa.

\section{REFERÊNCIAS}

1 Brasil. Ministério da Saúde. Sífilis: estratégias para diagnóstico no Brasil. Brasília: Ministério da Saúde, Coordenação de Doenças Sexualmente Transmissíveis e Aids; $2010 a$.

2 Avelleira JCR, Bottino G. Sífilis: diagnóstico, tratamento e controle. An. Bras. Dermatol. [Internet]. 2006 Mar [citado 2019 Nov 15] ; 81( 2 ): 111-126. Disponível em: <http://www.scielo.br/pdf/abd/v81n2/v81n02a02.pdf>.

3 Araújo LM, Reis KCT, Baldo G, Modesto CMS, Oliveira HC. Guia prático em abordagem sindrômica: prática baseada em evidências: sífilis. Cuiabá; 2017.

4 Brasil. Ministério da Saúde. Secretaria de Vigilância em Saúde. Departamento de Vigilância Epidemiológica. Doenças infecciosas e parasitárias: guia de bolso. 8. ed. rev. Brasília: Ministério da Saúde; 2010b.

5 Brasil. Ministério da Saúde. Boletim Epidemiológico Sífilis. 2016a; 47(35.

6 Brasil. Ministério da Saúde. Portaria $n^{\circ} 2.472$, de 31 de agosto de 2010. Define as terminologias adotadas em legislação nacional, conforme disposto no Regulamento Sanitário Internacional 2005 (RSI 2005), a relação de doenças, agravos e eventos em saúde pública de notificação compulsória em todo o território nacional e 
estabelecer fluxo, critérios, responsabilidades e atribuições aos profissionais e serviços de saúde. [portaria na internet]. [acesso em 2019 mar. 20]. Disponível em: bvsms. saude.gov.br/bvs/saudelegis/gm/2010/prt2472_31_08_2010.html.

7 Who. Guidelines for the treatment of treponema pallidum (syphilis). Geneva: World Health Organization; 2016. [acesso em 2020 abr. 09]. Disponível em: https://www.who.int/reproductivehealth/publications/rtis/syphilis-treatmentguidelines/en/.

8 Brasil. Ministério da Saúde. Secretaria de Vigilância em Saúde. Departamento de Vigilância, Prevenção e Controle das Doenças Sexualmente Transmissíveis, Aids e Hepatites Virais. Manual Técnico para Diagnóstico da Sífilis. Brasília: Ministério da Saúde; 2016c.

9 Cofen. Resolução Cofen $n^{\circ}$ 544/2017. [acesso em 2019 nov. 19]. Disponível em:http://www.cofen.gov.br/resoluo-cofen-1591993_4241.html.

10 Monteiro ACM, Rodrigues BMRD, Pacheco STA, Pimenta LS. A atuação do enfermeiro junto à criança com câncer: cuidados paliativos. Rev. enferm. UERJ. [internet] 2014 nov./dez. [citado em 2019 mar. 20]; 22(6): 828-833. Doi: http://dx.doi.org/10.12957/reuerj.2014.15665

11 Barbosa TLA, Gomes LMX, Holzmann APF, Paula AMB, Haikal DS. Aconselhamento em doenças sexualmente transmissíveis na atenção primária: percepção e prática profissional. Acta paul. enferm. [Internet]. 2015 Dec [citado 2019 Mar 20]; 28(6): 531-538. Doi: https://doi.org/10.1590/1982-0194201500089.

12 Brasil. Ministério da Saúde. Comissão Nacional de Incorporação de Tecnologias do SUS. Protocolo clínico e diretrizes terapêuticas infecções sexualmente transmissíveis [Internet]. Brasília: CONITEC; 2015 [citado em 2015 jun. 22]. Disponível em: http://conitec.gov.br/images/Consultas/Relatorios/2015/ Relatorio_PCDT_IST_CP.pdf

13 Brasil. Ministério da Saúde. Boletim epidemiológico: sífilis. Brasília: Ministério da Saúde; 2018.

14 Brasil. Ministério da Saúde. Secretaria de Atenção à Saúde. Departamento de Atenção Básica. Rastreamento. Brasília: Ministério da Saúde; 2010d.

15 Sousa CP. Adolescentes: maior vulnerabilidade às IST/AIDS/RETEP. Rev. Tendên. Enferm. Profis. 2017; 9(4): 2289-2295.

16 Soares ES, Carvalho EM, Lima KTLL. Incidência de sífilis adquirida em uma cidade da microrregião do sudoeste baiano. Rev. Bras. Analises Clínicas. [internet] 2019 [citado em 2020 mar. 31] ;51(2):115-19. Disponível em: http://www.rbac.org.br/ artigos/incidencia-de-sifilis-adquirida-em-uma-cidade-da-microrregiao-do-sudoestebaiano. 
17 Brasil. Ministério da Saúde. Secretaria de Vigilância em Saúde. Departamento de Vigilância, Prevenção e Controle das Doenças Sexualmente Transmissíveis, Aids e Hepatites Virais. Manual Técnico para Diagnóstico da Sífilis. - Brasília: Ministério da Saúde, 2016b.

18 Komka MR, Lago EG. Sífilis congênita: notificação e realidade. Sci méd. 2007;17(4): 205-211.

19 Dantas LA, Jerônimo SHNM, Teixeira GA, Lopes TRG, Cassiano NA, Carvalho JBL. Perfil epidemiológico de sífilis adquirida diagnosticada y notificada en hospital universitario materno infantil. Enferm. glob. [internet] 2017 [citado em 2020 mar. 31];16(46 ):217-245. Disponivel em: http://scielo.isciii.es/scielo.php? script=sci_arttext\&pid=S1695-61412017000200217\&lng=es

20 Cavalcante EGF, Miranda MCC, Carvalho AZFHT, Lima ICV, Galvão MTG. Partner notification for sexually transmitted infections and perception of notified partners. Rev. esc. enferm. USP [Internet]. 2016 June [citado em 2020 mar. 20]; 50(3): 450-457. Doi: http://dx.doi.org/10.1590/S0080-623420160000400011

21 Padovani C, Oliveira RR, Pelloso SM. Syphilis in during pregnancy: association of maternal and perinatal characteristics in a region of southern Brazil. Rev. Latino-Am. Enfermagem [Internet]. 2018 [citado 2020 mar. 20]; 26: e3019. Doi: http://dx.doi.org/10.1590/1518-8345.2305.3019.

22 Motta IA, Delfino IRS, Santos LV, Morita MO, Gomes RGD, Martins TPS et al. Congenital syphilis: why is its prevalence still so high. Rev Med Minas Gerais [internet] 2018 [citado em 2020 mar. 20];28 (Supl.6): e-S280610. Doi: http://dx.doi.org/10.5935/2238-3182.20180102.

23 Brasil. Ministério da Saúde. Secretaria de Atenção à Saúde. Departamento de Atenção Básica. Acolhimento à demanda espontânea. Brasília: Ministério da Saúde; 2013.

24 Miranda LCV. Sistematização da assistência de enfermagem na atenção primária à saúde: um relato de experiência. Rev enferm UFPE. 2013 jan.;7(1):295-301.

Artigo recebido em: 10/12/2019

Artigo aprovado em: 05/07/2020

Artigo publicado em: 06/07/2020 